\title{
Clustering and mobility of hard rods in a quasicrystalline substrate potential
}

Cite as: J. Chem. Phys. 137, 224705 (2012); https://doi.org/10.1063/1.4769839

Submitted: 21 September 2012 - Accepted: 09 November 2012 • Published Online: 14 December 2012

Philipp Kählitz, Martin Schoen and Holger Stark



View Online

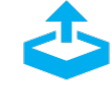

\section{ARTICLES YOU MAY BE INTERESTED IN}

Phase ordering of hard needles on a quasicrystalline substrate

The Journal of Chemical Physics 136, 174705 (2012); https://doi.org/10.1063/1.4711086

Formation of dodecagonal quasicrystals in two-dimensional systems of patchy particles

The Journal of Chemical Physics 136, 054904 (2012); https://doi.org/10.1063/1.3679653

Liquid to quasicrystal transition in bilayer water

The Journal of Chemical Physics 133, 154516 (2010); https://doi.org/10.1063/1.3499323

The Journal

SPECIAL TOPIC: Low-Dimensional

of Chemical Physics Materials for Quantum Information Science 


\title{
Clustering and mobility of hard rods in a quasicrystalline substrate potential
}

\author{
Philipp Kählitz, ${ }^{1, a)}$ Martin Schoen, ${ }^{2, b)}$ and Holger Stark ${ }^{1, c)}$ \\ ${ }^{1}$ Institut für Theoretische Physik, Technische Universität Berlin, Hardenbergstr. 36, D-10623 Berlin, Germany \\ ${ }^{2}$ Stranski-Lab. für Physikalische und Theoretische Chemie, Fakultät für Mathematik und Naturwissenschaften, \\ Str. des 17. Juni 135, 10623 Berlin, Germany and Department of Chemical and Biomolecular Engineering, \\ North Carolina State University, Campus Box 7905, Raleigh, North Carolina 27695-7905, USA
}

(Received 21 September 2012; accepted 9 November 2012; published online 14 December 2012)

\begin{abstract}
Recently, we have studied the self-assembly of hard needles in a quasicrystalline substrate potential with decagonal symmetry [P. Kählitz and H. Stark, J. Chem. Phys. 136, 174705 (2012)]. We have identified new structure formation using Monte Carlo simulations. However, hard needles have a zero width. To investigate how the excluded volume of rod-shaped particles influences their phase ordering, we extend here our studies to spherocylinders. We determine phase diagrams and plot them in the relevant variables, strength of substrate potential versus area fraction. At increasing area fraction $\eta$ short rods form clusters that ultimately destroy directional ordering along the decagonal symmetry directions while surface-induced positional order exists for all $\eta$. In contrast, long rods show directional order in the whole density range. However, at high area fractions they assemble into compact clusters which destroy positional ordering. Finally, we also study the rod mobility using the kinetic Monte Carlo method and discuss an unexpected mobility enhancement with increasing density. All these features crucially depend on the non-zero excluded volume of the spherocylinders. (C) 2012 American Institute of Physics. [http://dx.doi.org/10.1063/1.4769839]
\end{abstract}

\section{INTRODUCTION}

Creating new structures through self-assembly of small units is a promising route for fabricating nanostructured functional systems. Circa one decade ago, research has been initiated to study the self-assembly of atoms on the surface of quasicrystals. ${ }^{2}$ It was found that the atomic film either adopts the quasicrystalline structure and thereby may be extended into the third dimension or that it shows the periodic close-packed structure the adsorbed atoms prefer to form. ${ }^{2-16}$ Quasicrystals are nonperiodic in space but nevertheless exhibit long-range positional order ${ }^{17-21}$ which gives rise to interesting material properties. ${ }^{22}$ For example, the friction coefficient of a quasicrystalline surface is very low. ${ }^{23}$ So it is of interest to find methods to fabricate artificial quasicrystals, to understand how lubricants behave close to quasicrystal surfaces and ultimately influence their friction coefficients, or simply to identify novel structure formation. In this article, we will extend our work on the self-assembly of hard needles in a quasicrystalline substrate potential ${ }^{1}$ and investigate hard rods with a finite width. We will demonstrate how this influences the structure formation of elongated particles.

Instead of simple atoms recent work has also investigated monolayers of hydrocarbons on quasicrystal surfaces using very detailed surface potentials from the embeddedatom method. ${ }^{16,24,25}$ In parallel, one of the present authors

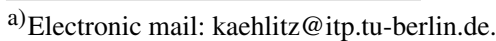

b) Electronic mail: martin.schoen@tu-berlin.de.

c)Electronic mail: holger.stark@tu-berlin.de.
}

(H.S.) was involved in studying simple model atoms such as colloidal particles in a quasicrystalline substrate potential. Indeed, besides showing hexagonal and quasicrystalline ordering, these systems helped to identify an unexpected Archimedian-like tiling and 20-fold bond-orientational order when particle-particle and particle-substrate interactions are comparable..$^{26-33}$ They are also useful to explore dynamic properties of quasicrystals such as the diffusion of particles in a quasicrystalline environment ${ }^{34}$ and their phasonic degree of freedom. ${ }^{35}$

In Ref. 1 we extended the study of ordering on quasicrystalline substrates to the simplest form of an elongated particle, a hard needle. The orientational degree of freedom leads to interesting directional order along the decagonal symmetry directions of the substrate potential in combination with pronounced cluster formation. This ordering competes with the nematic phase which needles form at sufficiently large densities. However, needles have artificial properties. Their excluded volume is zero or at most very small due to thermal fluctuations and many of them can occupy the same potential minima which results in very compact clusters. ${ }^{1}$ In this article we choose spherocylinders to model hard rods with finite width. They consist of cylinders and two hemispheres placed at both ends as caps. We use spherocylinders to mimic the rodshaped form of real systems such as alkane molecules or more rigid elongated organic molecules which might be placed on the surface of an atomic quasicrystal. Towards the micrometer scale hard rods are well approximated by the mosaic tobacco viruses ${ }^{36-39}$ and natural clay or Boehmite rods, ${ }^{40-42}$ which react on the quasicrystalline interference pattern of laser beams. ${ }^{26}$ 
The phase behavior of hard spherocylinders confined to two dimensions was investigated in simulations first by Bates and Frenkel. ${ }^{43}$ They observed that phase ordering depends on the aspect ratio of the rods, $L / D$. For aspect ratios below $L / D$ $\approx 7$, the rods exhibit only an isotropic phase at low densities and a solid phase at high densities. Spherocylinders with higher aspect ratios show an isotropic-quasi-nematic phase transition. In the quasi-nematic phase the orientational correlations decay algebraically as in the hard-needle system. ${ }^{44}$ Therefore, the nematic order parameter strongly depends on system size and vanishes in the thermodynamic limit. Our rod system is sufficiently small for nematic order to occur which is fully correlated over the whole system. At larger densities rods with a high aspect ratio also show a transition to a solid phase which looks almost smectic but further properties of the phase are not known. ${ }^{43}$ In our simulations we stay in a range of area fractions where the solid does not occur.

In this article we demonstrate how the excluded volume of spherocylinders determines the phase behavior of rods on a decagonal substrate. We concentrate on an aspect ratio of $L / D$ $=10$, where they form a quasi-nematic phase. To illustrate the basic features of our system, we introduce a short and a long rod that match the characteristic dimension of the substrate potential. The rods exhibit characteristic directional and positional order induced by the substrate. For increasing area fraction clusters start to form and determine the observed structures. We explain why at large area fractions directional order of short rods vanishes while for long rods positional order is suppressed. Furthermore, we show how the decagonal substrate potential influences the mobility of the rods and discuss an unexpected mobility enhancement of short rods with increasing area fraction. As we will explain in detail, all these features strongly depend on the non-zero width of the rods and, therefore, are not observed in a system of hard needles with zero width. This demonstrates that there are clear qualitative differences between a hard-needle and a hard-rod system.

The article is organized as follows. We introduce the model of the hard spherocylinder and the substrate potential in Sec. II, where we also explain details of the conventional and kinetic Monte Carlo simulations. In Sec. III we discuss the simulation results for the short- and long-rod system, separately. We present the phase diagram and then discuss more details of the phase ordering. Finally, we show the results from the kinetic Monte Carlo simulations for the mobility of the rods. We close the article with a summary and conclusions in Sec. IV.

\section{MODEL}

\section{A. Hard spherocylinder and order parameters}

We model the rods which we place in the decagonal substrate potential by hard spherocylinders. They interact through hard core repulsion only and are not allowed to overlap. Throughout the article, we use an aspect ratio of rod length to diameter of $L / D=10$ to exemplify the ordering of elongated particles. The spherocylinder is illustrated in Fig. 1.

We use three order parameters to classify ordering of the rods in the substrate potential. From literature it is well known

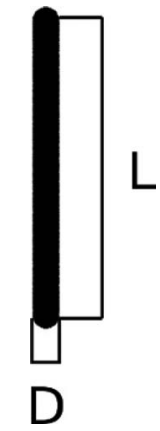

FIG. 1. Parameters of a spherocylinder: length $L$ and diameter $D$.

that spherocylinders with aspect ratio 10 form a quasi-nematic phase in two dimensions without any substrate potential. ${ }^{43}$ To identify nematic ordering, we calculate the orientational tensor order parameter with zero trace for a configuration of $N$ rods,

$$
Q_{a b}=N^{-1} \sum_{i=1}^{N}\left[2 u_{a}(i) u_{b}(i)-\delta_{a b}\right],
$$

where the unit vector $\vec{u}(i)=\left[u_{1}(i), u_{2}(i)\right]$ indicates the direction of rod $i$. The two eigenvalues of $Q_{a b}, Q$, and $-Q$ add up to zero and the nematic order parameter is the ensemble average $S=\langle Q\rangle$ over the positive eigenvalue of $Q_{a b}{ }^{44}$

The decagonal substrate potential exhibits 10 symmetry directions. To quantify how well the rods align along them, we introduce the $m$-fold directional order parameter,

$$
\Phi_{m}=\left\langle\varphi_{m}\right\rangle \quad \text { with } \quad \varphi_{m}=N^{-1}\left|\sum_{j=1}^{N} e^{i m \alpha_{j}}\right|,
$$

where $\alpha_{j}$ is the angle of rod $j$ with respect to an arbitrary axis. In our case, we determine $\Phi_{10}$ to measure decagonal directional ordering. We also use the phase angle of the complex sum in Eq. (2) to identify characteristic directions of the rods.

We will illustrate positional order of the rod centers by the two-dimensional pair correlation function. In addition, we introduce the $m$-fold bond-orientational order parameter,

$$
\Psi_{m}=\left\langle\psi_{m}\right\rangle \text { with } \psi_{m}=N^{-1}\left|\sum_{j=1}^{N} n_{j}^{-1} \sum_{k=1}^{n_{j}} e^{i m \omega_{j k}}\right|,
$$

where $\omega_{j k}$ is the angle of the bond between particle $j$ and one of its $n_{j}$ nearest neighbors $k$ with respect to an arbitrary reference direction. The nearest neighbors are determined with the help of a Voronoi tessellation. ${ }^{45,46}$ At increasing potential strength the rods form clusters. We define a cluster by the condition that all nearest neighbors $j$ of each particle $i$ within a cluster stay below a maximum distance, $\left|\vec{r}_{i}-\vec{r}_{j}\right|<4 D$, and have a maximum difference in their orientations, $\left|\alpha_{i}-\alpha_{j}\right|$ $<0.2$. We checked that the cluster definition is robust against variations of these limits. For each cluster we then define the position $\vec{r}_{C}$ of its center of mass and an angle $\alpha_{C}$ for its orientation. Finally, we introduce the cluster bond-orientational order parameter $\Psi_{C m}$ as before. The bond-orientational order parameter is very sensitive to deviations from an ideal positional order which in our case is induced by the decagonal substrate potential. Therefore, we will monitor 10- and 
20 -fold bond-orientational order of rods and clusters. We name the bond order after the order parameter with the highest value.

From our work on hard needles, we know that fluctuations of the order parameters help to identify phase transitions in the rod system. ${ }^{1}$ They become maximal at a phase transition and even show critical behavior when the transition is second order ${ }^{47}$ One quantifies order parameter fluctuations by their variances also known as susceptibilities. ${ }^{48,49}$ In our case the relevant susceptibilities for orientational, directional, and bond-orientational order are, respectively,

$$
\begin{aligned}
& \chi_{S}=\beta N\left(\left\langle Q^{2}\right\rangle-S^{2}\right), \\
& \chi_{\Phi}=\beta N\left(\left\langle\varphi_{m}^{2}\right\rangle-\Phi_{m}^{2}\right), \\
& \chi_{\Psi}=\beta N\left(\left|\psi_{m}^{2}\right\rangle-\Psi_{m}^{2}\right) .
\end{aligned}
$$

In the simulations we find the maxima of $\chi_{S}$ and $\chi_{\Phi}$ at an approximate value of 0.2 for the respective order parameters independent of the system parameters. We therefore define the onset of nematic and decagonal directional order at $S>0.2$ and $\Phi_{10}>0.2$, respectively. Maximal fluctuations in the bond-orientational order parameter occur already at about $\Psi_{m} \approx 0.1$, so we identify bond order for $\Psi>0.1$.

\section{B. Substrate potential}

We use the same decagonal substrate potential as in our study on hard needles. ${ }^{1}$ It can be realized by the interference pattern of five laser beams and was used to study colloidal ordering both in experiments ${ }^{26}$ and in theory. ${ }^{27,28,34}$ We define the decagonal potential

$$
V(\vec{r})=-\frac{V_{0}}{25} \sum_{j=0}^{4} \sum_{i=0}^{4} \cos \left[\left(\vec{G}_{i}-\vec{G}_{j}\right) \cdot \vec{r}\right],
$$

where the five wave vectors $\vec{G}_{i}$ point to the vertices of a regular pentagon as indicated in Fig. 2, left starting with $\vec{G}_{0}$ $=\left(0,2 \pi / a_{V}\right)$. Figure 2, right shows the color-coded decagonal substrate potential and the characteristic length scale $a_{V}$ $=2 \pi /\left|\vec{G}_{i}\right|$. In the following we give all lengths in units of $a_{V}$. We characterize the strength of the substrate potential by the parameter $V_{0}$, which is the depth of the deepest minima located at the origin, and always refer $V_{0}$ to the thermal energy $k T$, where $k$ is the Boltzmann constant and $T$ is temperature.

We define the potential energy of a rod interacting with the substrate potential by averaging $V(\vec{r})$ along the full rod length which leads to a new interaction potential that depends on the center-of-mass position $\vec{r}$ and the orientation $\vec{u}$ of the rod:

$$
V_{R}(\vec{r}, \vec{u})=\frac{1}{L} \int_{-1 / 2}^{1 / 2} V(\vec{r}+l L \vec{u}) d l .
$$

The properties of the potential $V_{R}(\vec{r}, \vec{u})$ clearly depend on the rod length $L$. Placed on the substrate potential $V(\vec{r})$, the rods assume an energy minimum when they connect potential minima as illustrated in Fig. 2, right. These minima define straight lines that are oriented along the ten symmetry directions of

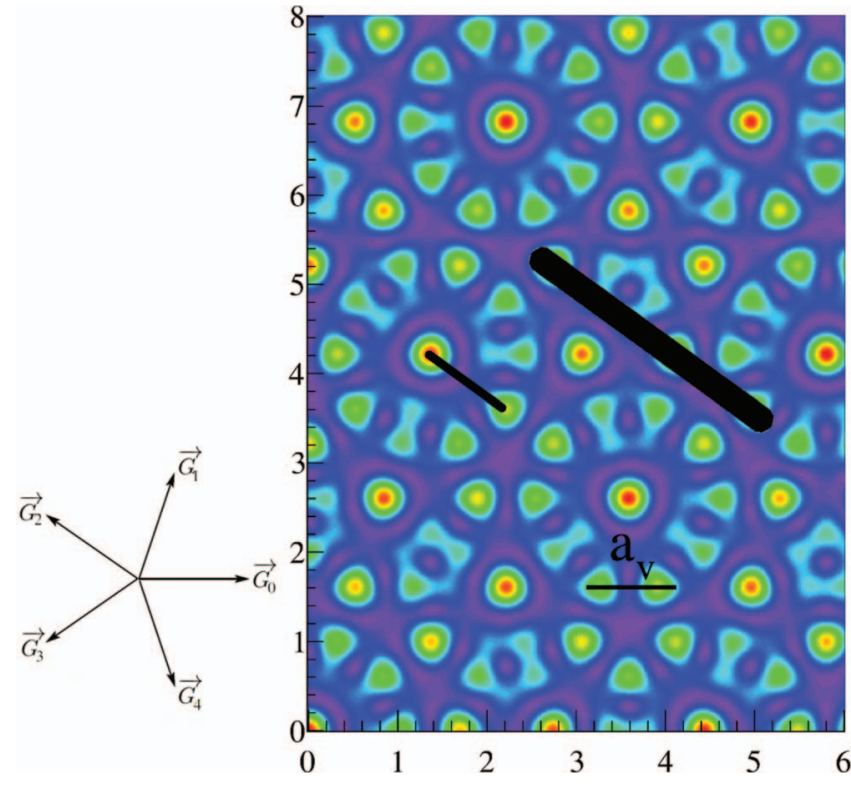

FIG. 2. Left: Wave vectors pointing to the vertices of a pentagon are used to set up the substrate potential in Eq. (7) with decagonal symmetry. Right: Color-coded decagonal substrate potential with the short and long rod. The characteristic length $a_{V}$ is indicated.

the decagonal substrate potential. Therefore, the rods preferentially orient themselves along these decagonal directions. The distances between the lines define Fibonacci sequences or one-dimensional quasiperiodic patterns. They were important for analyzing the needle system in Ref. 1 but are not so relevant for the rod system of the current work.

We checked that the basic features of our rod system can be evaluated by concentrating on two rod lengths [see Fig. 2, right]. We introduce short rods with length $L=1 a_{V}$ and long rods with length $L=3 a_{V}$. Since for both rods we choose an aspect ratio of ten, the long rods are also thicker and, therefore, completely occupy a minimum in the substrate potential.

\section{Simulation methods}

\section{Monte Carlo simulation}

To study the phase behavior of the hard rod system, we use a Monte Carlo NVT algorithm with periodic boundary conditions. ${ }^{1,50,51}$ We implement periodic boundary conditions for the aperiodic substrate potential and minimize the discontinuities at the boundaries of the simulation box by choosing special box sizes. Following our earlier work ${ }^{1}$ and Ref. 46, the rectangular simulation box has edge lengths $X=2 n a_{V}$ and $Y=m a_{V} / \sin (\pi / 5)$, where $n$ and $m$ are Fibonacci numbers. Different particle densities are realized by varying the particle number. In concrete, for the short-rod system we use a box size $(n, m)=(8,13)$ and a particle number between 100 and 637 to realize low densities and a box size $(n, m)=(5,8)$ with a particle number between 245 and 776 to realize high densities. The long-rod system is simulated in a box with $(n, m)=(34,55)$ and particle numbers varying between 229 and 1431. All particle densities are quantified by the area fraction $\eta$ which the rods occupy relative to the total area. 
Each Monte Carlo cycle of one particle consists of one rotational and one translational step. The acceptance rate for every step is adjusted to 0.5 by varying the maximum rotation angle and the maximum particle displacement. A complete Monte Carlo sweep is performed with $N$ Monte Carlo cycles for randomly chosen particles where $N$ is the total particle number. During equilibrating the system, about every 100th step we set the value for the maximum displacement to $3 L$ and the maximum rotation angle to $180^{\circ}$. Such sweeps have a very low acceptance ratio but they help the rod system to resolve locked states. To equilibrate the system, we use at least $10^{6}$ Monte Carlo sweeps for the low densities and 3 $\times 10^{6}$ sweeps for the high densities. We employ two different rod configurations as initial conditions. In the first we choose both the positions and orientations of the rods randomly, in the second all the rods are aligned into a common but arbitrarily chosen direction. With these two initial conditions we check whether the system is able to establish or remain in a quasi-nematic phase. For the short-rod system we perform at least ten independent simulation runs at every density and potential strength. For the long-rod systems the number of runs is at least four.

\section{Kinetic Monte Carlo simulation}

One can map the Monte Carlo step on a time step and then study the temporal evolution and dynamical properties of a system equivalent to a Brownian dynamics simulation in the overdamped limit. ${ }^{52}$ The resulting kinetic Monte Carlo method has been tested for spherical colloids. ${ }^{53}$ Recent studies refined the algorithm to treat particles with orientational degrees of freedom such as a spherical colloid with an intrinsic direction ${ }^{54}$ and spherocylinders which also exhibit anisotropic diffusion..$^{55}$

To map the Monte Carlo step with maximum displacement $r_{0}$ and acceptance ratio $A$ on the time step of a Brownian dynamics simulation with diffusion coefficient $D_{0}$, one introduces

$$
\Delta t_{0}=\frac{r_{0}^{2} A}{6 D_{0}} .
$$

This procedure is straightforward for the isotropic diffusion of a spherical particle. However, a rod has different diffusion coefficients parallel and perpendicular to the rod axis and also performs rotational diffusion. Furthermore, the acceptance ratios for the different Monte Carlo steps within a Monte Carlo cycle can be different. Equation (9) then introduces three different time steps $\Delta t_{\|}, \Delta t_{\perp}$, and $\Delta t_{R}$ for one cycle. To be consistent, they have to be the same. We therefore use as reference time step the diffusion time parallel to the rod axis, $\Delta t_{0}=\Delta t_{\|}=r_{\|}^{2} A_{\|} /\left(6 D_{\|}\right)$, and adjust the maximum step sizes for the other degrees of freedom so that $\Delta t_{\|}$ $=\Delta t_{\perp}=\Delta t_{R}$. This procedure results in the maximum step sizes,

$$
r_{\perp}=\sqrt{\frac{A_{\|} D_{\perp}}{A_{\perp} D_{\|}}} r_{\|} \quad \text { and } \quad r_{R}=\sqrt{\frac{A_{\|} D_{R}}{A_{R} D_{\|}}} r_{\|} .
$$

The step size $r_{\|}$should be well below the smallest characteristic length scale one wants to resolve. In our case, it is given by the diameter of $\operatorname{rod} D$. We therefore set $r_{\|}<0.1 D$ but also verified that $r_{\|}<0.01 D$ does not change our simulation results. Our choice of $r_{\|}<0.1 D$ is well suited to resolve the dynamics of the rod on a time scale it needs to diffuse a diameter $D$.

When we perform our simulations, we use the normal Monte Carlo scheme to equilibrate the system. Then we start the kinetic Monte Carlo scheme where we rescale the maximum step sizes as explained above. One Monte Carlo sweep goes consecutively through all the particles to avoid unphysical double moves or stops of one rod. The sequence of the particles is altered randomly between the sweeps.

Finally, we mention the diffusion coefficients we use to quantify the anisotropic diffusion of a spherocylinder: ${ }^{56}$

$$
\begin{gathered}
D_{\|}=\frac{D_{0}}{2 \pi}\left(\ln p-0.207+0.980 / p-0.133 / p^{2}\right), \\
D_{\perp}=\frac{D_{0}}{4 \pi}\left(\ln p+0.839+0.185 / p+0.233 / p^{2}\right), \\
D_{R}=\frac{3 D_{0}}{\pi L^{2}}\left(\ln p-0.662+0.917 / p-0.050 / p^{2}\right) .
\end{gathered}
$$

where $D_{\|}$and $D_{\perp}$ are the respective diffusion constants parallel and perpendicular to the rod axis and $D_{R}$ is the rotational diffusion coefficient. The quantity $p=L / D+1=11$ is the anisotropy parameter of the rods and $D_{0}=k T / \eta_{S} L$, where $\eta_{S}$ is the shear viscosity of the fluid.

\section{RESULTS}

We first discuss the phase ordering of short and long spherocylinders in Secs. III A and III B, respectively. In Sec. III C we present our results on the mobility study using kinetic Monte-Carlo simulations.

\section{A. Short rods}

The phase diagram of the short rods in Fig. 3 exhibits several phases which differ by their bond-orientational, their directional, and orientational order. Below the horizontal black line at a potential strength $V_{0} \approx 30$, the system of spherocylinders displays the usual phase sequence isotropic-nematic with increasing area fraction $\eta$ which one observes without any substrate potential. With increasing $V_{0}$ the isotropicnematic phase transition shifts to larger $\eta$. Above the potential strength of $V_{0} \approx 30$ the phase diagram is divided into regions A-D with different bond-orientational order to be discussed below. In addition three characteristic density ranges exist. At very low area fractions of $\eta<0.12$ (regions A and B) the single rods display surface-induced bond-orientational order as well as pronounced directional order with non-zero order parameter $\Phi_{10}$. In the regime of intermediate area fractions $0.12<\eta<0.35$ (regions $\mathrm{C}$ and $\mathrm{D}$ ) the rods form well separated clusters under the influence of the surface potential. Now, the clusters display bond-orientational and directional order similar to the single rods in the dilute regime. At large area fractions of $\eta>0.35$ the clusters touch each other. While 


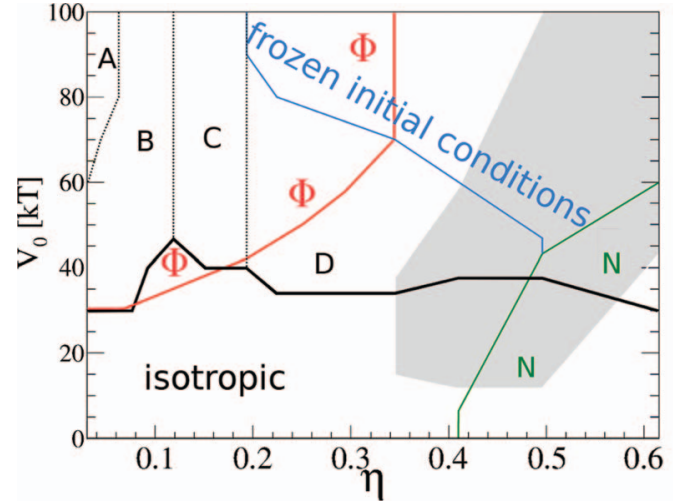

FIG. 3. Phase diagram for the short-rod system. Above the thick black line a bond-orientational order parameter $\Psi>0.1$ indicates positional order induced by the quasicrystalline substrate potential. The different regions are classified according to the most pronounced bond order: $\Psi_{20}$ in region A, $\Psi_{10}$ in $\mathrm{B}, \Psi_{C 20}$ in $\mathrm{C}$, and $\Psi_{C 10}$ in D. The red line marked with the symbol $\Phi$ denotes a region with a decagonal directional order parameter $\Phi_{10}>0.2$. Below the green line marked by the symbol $N$ nematic order with $S>0.2$ occurs. In the gray shaded region the preferred orientations of the rods lie between the symmetry directions of the substrate potential. Above the blue line named frozen initial conditions, the system keeps an initial nematic or isotropic order.

the directional order parameter falls below $\Phi_{10}=0.2$ (in contrast to the short-needle system studied in Ref. 1), still a delicate directional order is observable as we explain below. In particular, in the gray shaded region the preferred directions of the rods lie between the symmetry directions of the surface potential. Finally, at area fractions above $\eta=0.19$ and sufficiently large potential strength $V_{0}$, an initial isotropic state or nematic order remains after equilibration. We now discuss the different regions in more detail.

In Fig. 4 we plot bond-orientational order parameters for rods $\left(\Psi_{10}, \Psi_{20}\right)$ and the center of mass of rod clusters $\left(\Psi_{C 10}\right.$, $\left.\Psi_{C 20}\right)$. Below an area fraction of $\eta=0.12$ most rods are well separated from each other and only a few clusters exist. At very low area fractions $\eta<0.08$ and large potential strengths $V_{0}>60 k T$ a 20 -fold bond-orientational order dominates (region $\mathrm{A}$ in Fig. 3). It occurs since the system is so dilute that nearest neighbors also occupy minima which lie in directions between the 10 symmetry directions of the substrate poten-

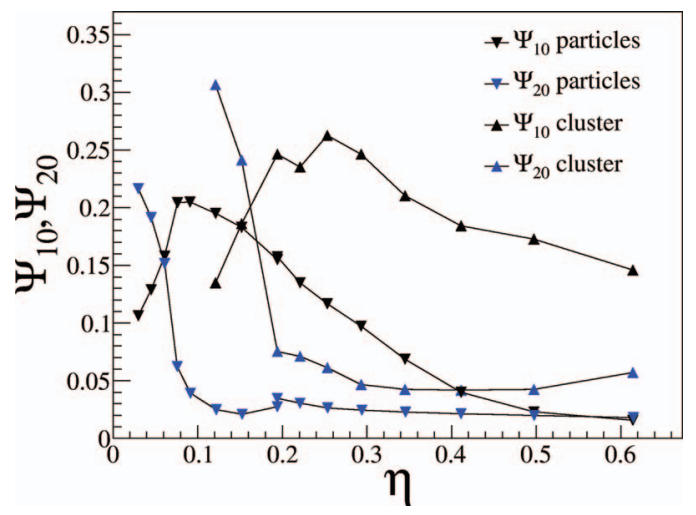

FIG. 4. Bond-orientational order parameters for rods $\left(\Psi_{10}, \Psi_{20}\right)$ and clusters of rods $\left(\Psi_{C 10}, \Psi_{C 20}\right)$ plotted versus area fraction at a potential strength of $V_{0}=80 k T$.
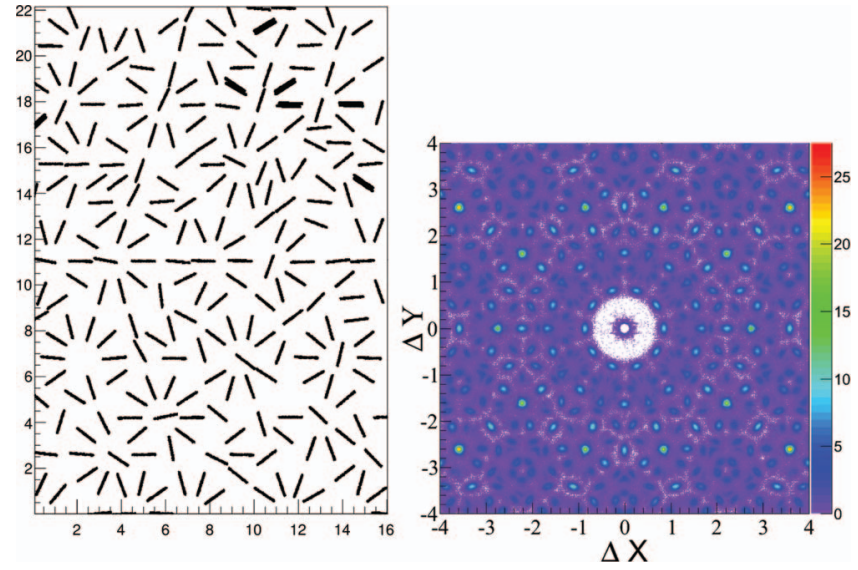

FIG. 5. Snapshot (left) and 2D pair correlation function (right) for the shortrod system at $V_{0}=100 k T$ and a low area fraction $\eta=0.08$ in region B.

tial. 10-fold bond-orientational order forms at lower potential strengths down to $V_{0}=40 k T$ and for area fractions up to $\eta=0.12$ (region B). For $\eta>0.08$ most of the minima in the rod potential $V_{R}$ are occupied and clusters of rods start to form. This is already visible in Fig. 5 at an area fraction $\eta$ $=0.08$. The rods form a pattern of decagonal flower structures and the sharp maxima in the pair correlation function indicate the long-range positional order with decagonal symmetry induced by the surface potential. At area fractions above $\eta$ $=0.12$ the number of rods well exceeds the number of deep minima in $V_{R}$. Within one cluster two or more rods start to occupy the same minimum in $V_{R}$ or, differently speaking, they connect two to three minima in the substrate potential $V(\vec{r})$. These clusters behave now like single rods in the very dilute regime. When the cluster density is low (region $\mathrm{C}$ in Fig. 3), they exhibit 20-fold bond-orientational order as indicated by $\Psi_{C 20}>\Psi_{C 10}$ in Fig. 4 in the range $\eta=0.12$ to 0.19 . Then, further increasing the area fraction $\eta 10$-fold bond-orientational order dominates in region $\mathrm{D}$ which extends to large densities. In Fig. 6(a) we show the snapshot of a rod system at $\eta=0.19$ and $V_{0}=100 k T$ together with the pair correlation function of the center of mass of the clusters. As in the single-rod case, the pair correlation function for the clusters shows sharp maxima. The bond-orientational order of the clusters decays quite slowly with increasing area fraction (Fig. 4). The 10-fold order parameter $\Psi_{C 10}$ stays above 0.1 for $V_{0}>40 k T$ and all simulated area fractions $\eta>0.19$. In Fig. 6(b) we show a high-density system with $\eta=0.5$. Even though the clusters are not separated from each other anymore, one can still identify flower-like structures formed by the densely packed clusters. In these structures, the clusters are oriented more along directions in between the symmetry directions of the substrate potential, which we will investigate further below. In contrast to the previous cases, the cluster pair correlation function now displays broader peaks which we attribute to the following observation. The clusters connect two to three minima of the substrate potential (for a schematic see Fig. 9). This creates a broad potential well for the rods in which they perform thermal motion resulting in the broadened peaks.

Figure 7 shows the decagonal directional order parameter $\Phi_{10}$ as a function of the potential strength $V_{0}$ for different 


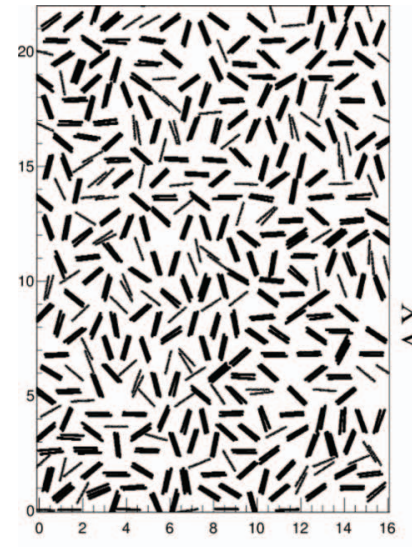

(a)
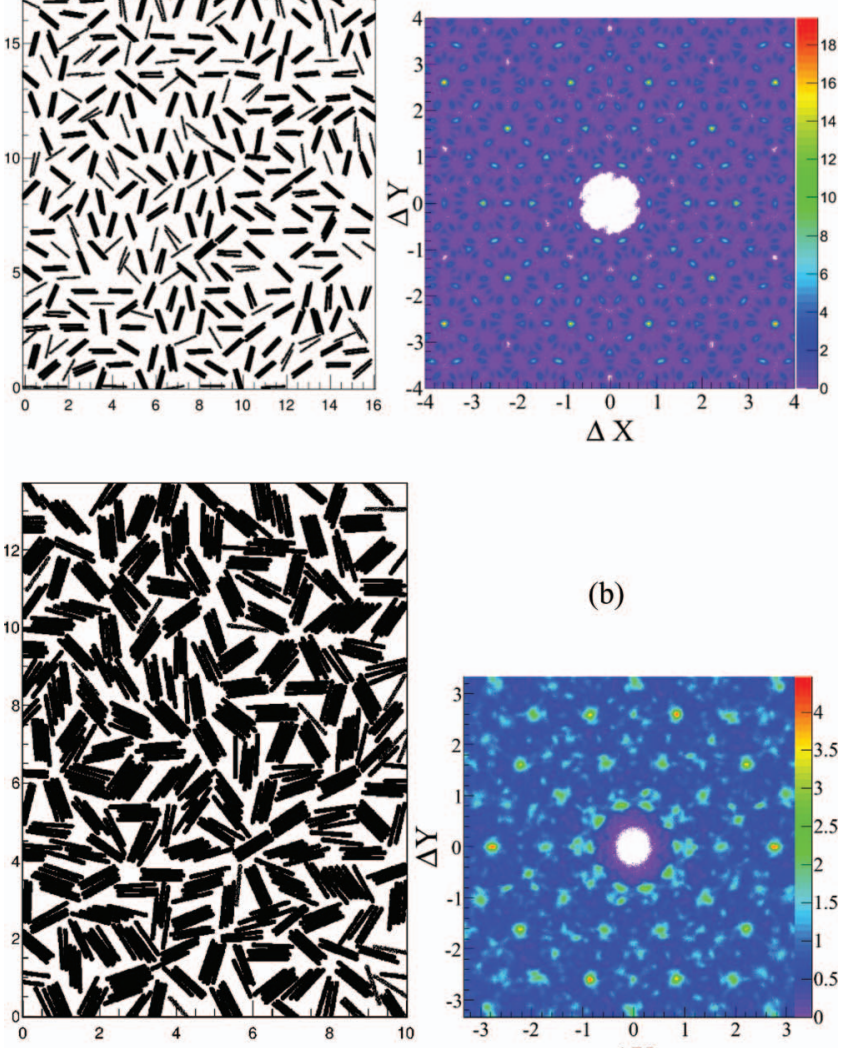

(b)

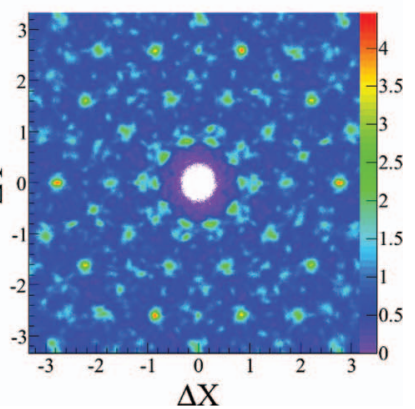

FIG. 6. Snapshots (left) and 2D pair correlation functions of the center of mass of the clusters (right) for the short-rod system at $V_{0}=100 \mathrm{kT}$ and (a) $\eta$ $=0.19$, and (b) $\eta=0.5$.

area fractions. With increasing $\eta$ decagonal directional order is reduced. Finally, at $\eta=0.42$ and larger area fractions the directional order parameter $\Phi_{10}$ always stays below 0.2 for all simulated potential strengths. This is quite different to what we observed in Ref. 1 . However, the substrate potential still affects the orientations of the rods. In Fig. 8 we plot their full directional distribution function $f(\alpha)$ for three different area fractions and potential strengths. At a low area fraction of $\eta$ $=0.19$ and low potential strength $V_{0}=26$ (isotropic phase),

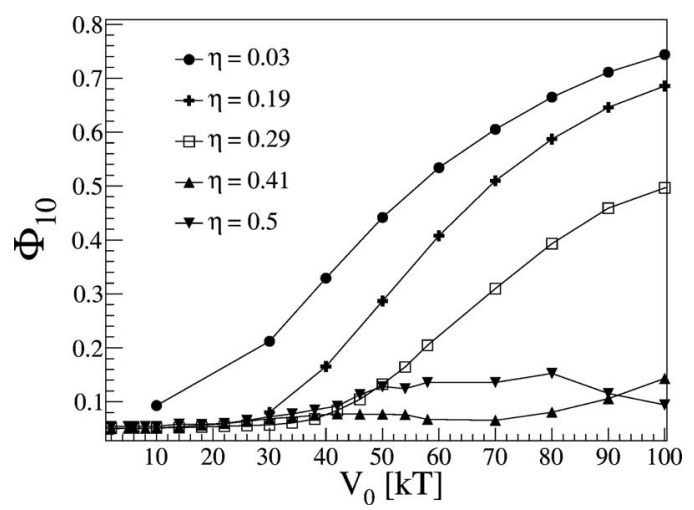

FIG. 7. Decagonal directional order parameter $\Phi_{10}$ plotted versus $V_{0}$ for different area fractions.
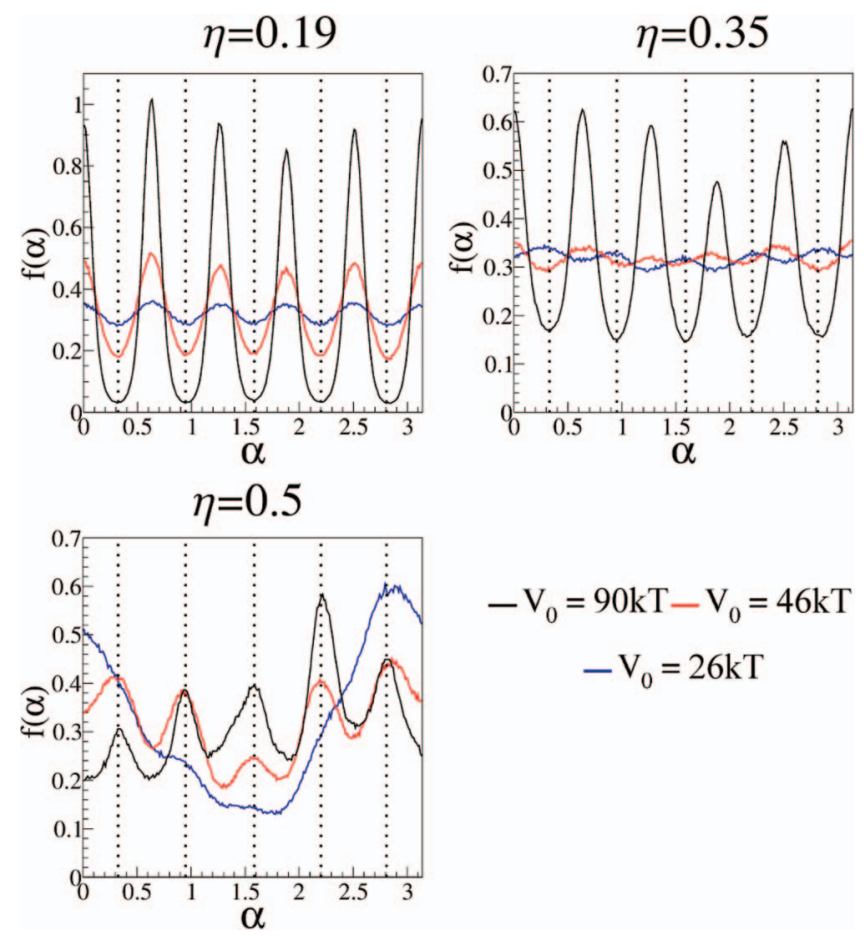

$-\mathrm{V}_{0}=90 \mathrm{kT}-\mathrm{V}_{0}=46 \mathrm{kT}$

$-\mathrm{V}_{0}=26 \mathrm{kT}$

FIG. 8. Directional distribution function $f(\alpha)$ for three area fractions $\eta$ and three potential strengths $V_{0}$.

the substrate potential only induces a small modulation of the isotropic distribution. The maxima coincide with the 10 symmetry directions of the decagonal substrate potential. The directional order parameter stays below $\Phi_{10}=0.1$. The difference between maxima and minima in $f(\alpha)$ grows with increasing $V_{0}$ until a directionally ordered phase with $\Phi_{10} \geq 0.2$ is established. The locations of the maxima and minima stay the same. This changes when the area fraction is increased. At $\eta=0.35$ and low potential strength $V_{0}=26 k T$, the preferred directions are located between the 10 symmetry directions. In the phase diagram of Fig. 3 the gray shaded region marks the parameter space where such a shift in the directions occurs. At the highest simulated potential strength $V_{0}=90 \mathrm{kT}$ the rod system exhibits directional order with $\Phi_{10}>0.2$ and the preferred directions agree again with the symmetry directions. In dense rod systems with a large area fraction the preferred directions of the rods remain shifted at large potential strengths $V_{0}$. We illustrate this in Fig. 8 for $\eta=0.5$. However, even at $V_{0}=90 \mathrm{kT}$, where the maxima are pronounced, does the directional order parameter stay below $\Phi_{10}=0.2$. Note that at a potential strength of $V_{0}=26 k T$ the rod system is in the nematic phase and the director aligns along one of the shifted preferred directions. These directions occur when the size of the rod clusters increases from small clusters with up to three rods per cluster to larger clusters. The schematic drawing of Fig. 9 illustrates the preferred directions. Single rods and clusters of up to three rods (Fig. 9, left) connect two deep minima of the substrate potential. However, clusters consisting of four rods and more (right) connect more shallow minima since they occupy more area which the deep minima cannot provide. So their preferred directions lie between the symmetry directions of the substrate potential. The mean energy of 

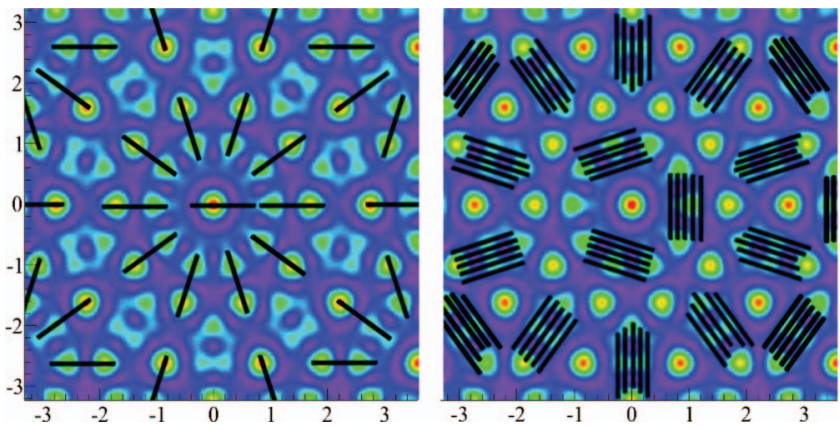

FIG. 9. Single rods and small clusters (left) occupy different positions and directions in the substrate potential than larger clusters (right) which gives rise to the gray shaded region in the phase diagram of Fig. 3 (schematic drawing).

a rod in a three-particle cluster is always below the respective value in larger clusters. So, in the high-density regime starting at $\eta=0.35$ the rod system consists of a mixture of small and big clusters with a rising fraction of big clusters at larger area fractions. Hence, there is no sharp transition from one set of preferred directions to the other set. Finally, we find it remarkable that the gray shaded region in Fig. 3 indicating the shifted preferred directions includes the isotropic and nematic phase, but also regions with bond-orientational order and with frozen initial conditions.

\section{B. Long rods}

Figure 10 shows the phase diagram of long rods which looks much simpler than the one for short rods. Below the potential strength $V_{0}=20$, one observes the typical isotropicnematic phase transition completely unaffected by the substrate potential. Above $V_{0}=20$, spherocylinders with length $3 a_{V}$ connect several minima of the surface potential (see Fig. 2) and therefore become mainly oriented along one of the symmetry directions. For this reason, one obtains directional order with decagonal symmetry that extends along the whole range of area fractions in contrast to the short-rod system but similar to the long-needle system in Ref. 1. Figure 11 demonstrates that the directional order parameter plotted versus $V_{0}$ only weakly depends on the area fraction $\eta$. Since the rods can slide to a certain degree along the minima, which they occupy,

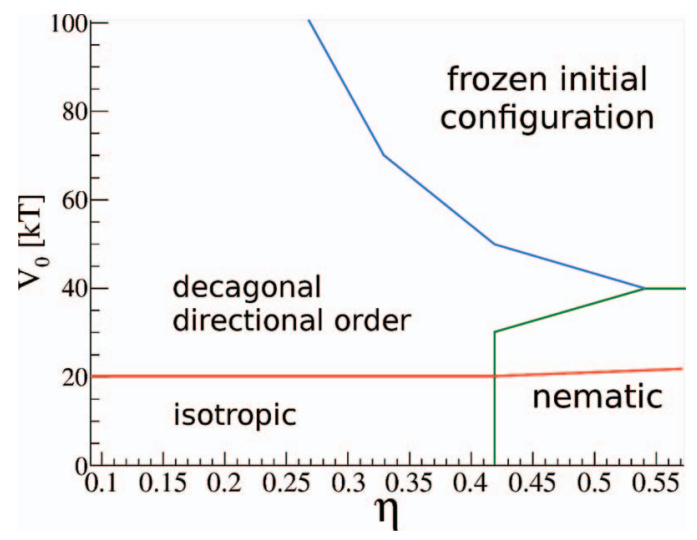

FIG. 10. Phase diagram of the long-rod system

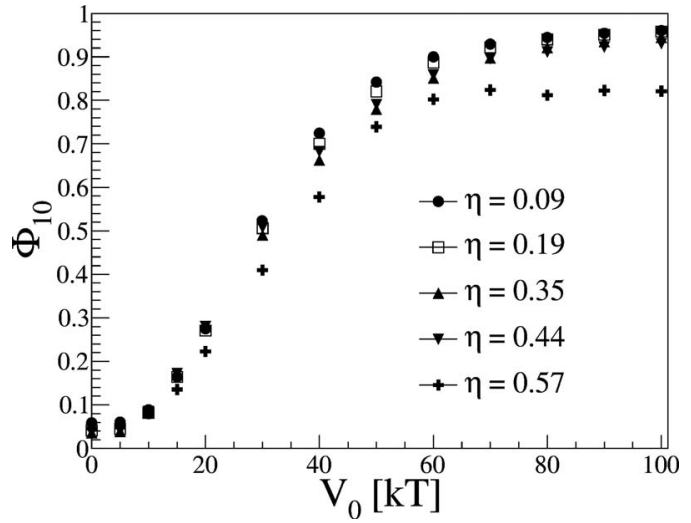

FIG. 11. Decagonal directional order parameter $\Phi_{10}$ plotted versus potential strength $V_{0}$ for different area fractions $\eta$.

the positional order is not sufficient to generate a pronounced bond-orientational order as observed for short rods. The bondorientational order parameter $\Psi$ is always below 0.1 . Nevertheless, as we will see below, in the pair correlation function one can identify preferred positions of the rods as dictated by the substrate potential. Furthermore, rod clusters that form at increasing area fraction show a delicate short-range order. As in a long-needle system, which we investigated in Ref. 1, the nematic phase extends into the region of decagonal directional order. Finally, at higher potential strengths the initial configuration, for example, a nematic order, can be frozen in.

We now study in more detail positional order with the help of the two-dimensional pair correlation function which we determine in the local frame of the rod. So we describe positional correlations of the rod's center of mass with respect to the coordinate system whose axes are parallel to the long and short axes of each individual rod, respectively. Figure 12(a) shows the snapshot of a low-density system at area fraction $\eta=0.19$. The pair correlation function in the local rod frame exhibits peaks according to the substrate potential but they are broadened due the motion of the rods. One also clearly recognizes a stripe pattern parallel to the local rod on which the peaks lie interrupted by blue bands with low positional correlations. They belong to the Fibonacci sequence, a one-dimensional quasiperiodic structure which one can assign to the substrate potential. ${ }^{1}$ However, our analysis shows that nearest-neighbor minima on this Fibonacci sequence are not occupied due to thermal motion of the rods so the pronounced stripes occur.

At increasing density clusters of rods form and positional order still exists. Now, the nearest-neighbor minima are filled up and the pronounced stripe pattern vanishes as indicated in Fig. 12(b). Unlike short rods, the long rods cannot cluster together in the same line of potential minima since their diameter approximately equals the width of the minima. Each rod is trapped in a different line of minima. In the snapshot of Fig. 12(b) one can clearly see spaces between parallel rods. Indeed the corresponding pair correlation function exhibits a correlation hole between the excluded volume of the central rod (in white color) and the nearest neighbors. As the relevant maxima indicate, the bond between nearest neighbors is not along their common short axis but points along the 


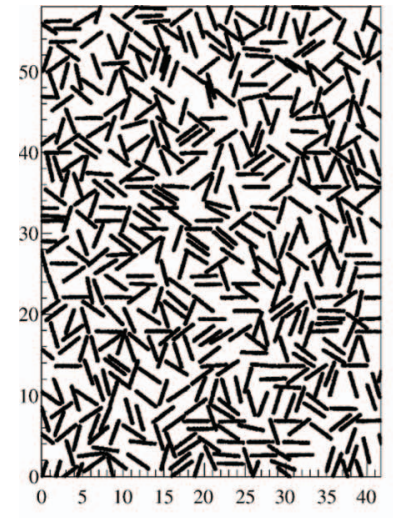

(a)
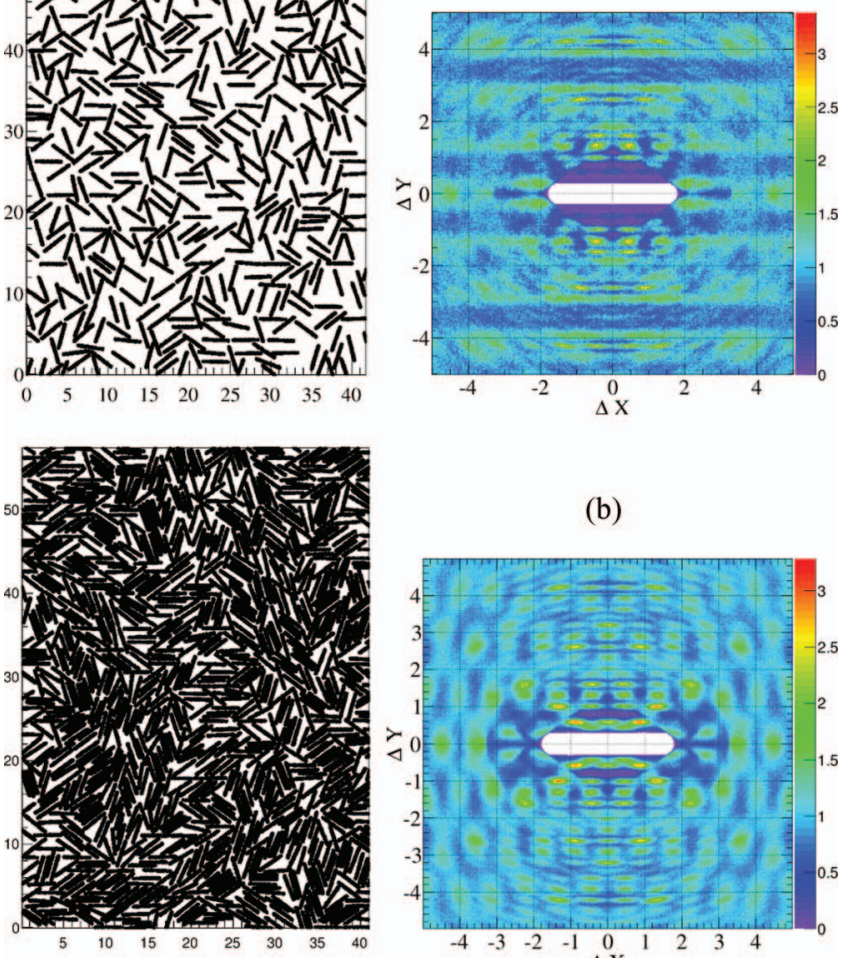

(b)
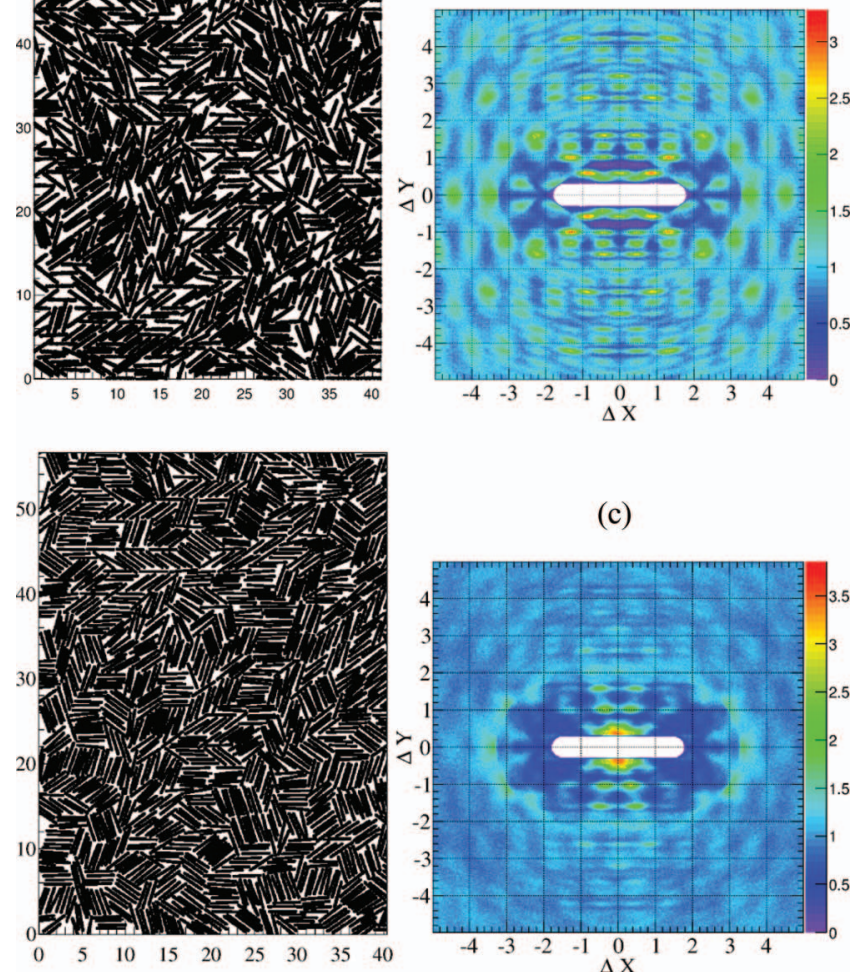

(c)

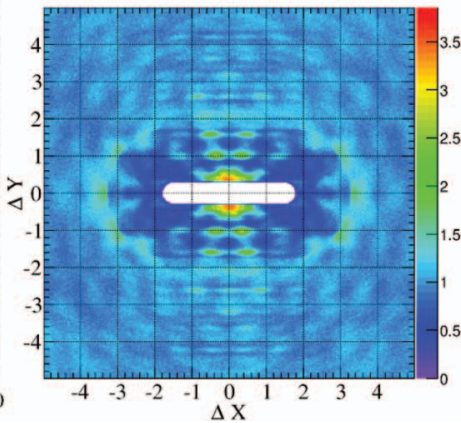

FIG. 12. Snapshots (left) and 2D pair correlation functions in the local rod frame (right) for the long-rod system at $V_{0}=60$ and (a) $\eta=0.19$, (b) $\eta$ $=0.44$, and (c) $\eta=0.57$.

symmetry directions of the potential at angles $\pm \pi / 5$ relative to the central rod. Hence, the clusters in the snapshot exhibit their typical rhombic shape. The distance of neighboring rods is the smallest distance of the Fibonacci sequences associated with the substrate potential. Compared to short rods, which at comparable area fraction [see Fig. 6(b)] form well separated clusters in a close side-by-side configuration, the behavior of long rods with the same aspect ratio is quite different.

Increasing the area fraction further, the whole system is compressed so that the rods start to touch each other. This is indicated in the pair correlation function of Fig. 12(c), where the nearest-neighbor maximum is located at the border of the excluded volume. Furthermore, the surface-induced positional order has vanished almost completely since the rods fill the whole space quite uniformly. This is in stark contrast to the long-needle system studied in Ref. 1. Partially, the rods form elongated clusters by ordering side by side and head to tail. Finally, decagonal directional order is still very high since the rods strictly align along the symmetry directions of the substrate potential.

\section{Kinetic Monte Carlo simulations}

We now present the results of our kinetic Monte Carlo simulations on the mobility of the rods both for single and multi-particle systems. Figure 13(a) shows the mean square displacement of a single short rod for different potential strengths. At $V_{0}=10 k T$ the mean square displacement is nearly the same as without substrate potential. With increasing $V_{0}$ the rod becomes trapped in a pair of deep potential minima and can only leave them by thermal activation. As a result, a subdiffusive regime develops before the rod performs normal diffusion. At $V_{0}=100 k T$ the rod stays trapped in its potential minima and the regime of normal diffusion is not reached within the simulation time. In Fig. 13(b) we plot the angular mean square displacement $\left\langle\varphi^{2}\right\rangle$ and the accumulated displacements parallel $\left(\left\langle r_{\|}^{2}\right\rangle\right)$ and perpendicular $\left(\left\langle r_{\perp}^{2}\right\rangle\right)$ to the


FIG. 13. (a) Mean square displacement $\left\langle r^{2}\right\rangle$ of a single short rod in units of $a_{V}^{2}$ for different potential strengths $V_{0}$. Time is measured in units of $a_{V}^{2} / D_{\|}$, where $D_{\|}$is the single-rod diffusion coefficient parallel to the rod axis [see Eq. (11)]. (b) Angular mean square displacement $\left\langle\varphi^{2}\right\rangle$ and accumulated mean square displacement parallel $\left(\left\langle r_{\|}^{2}\right\rangle\right)$ and perpendicular $\left(\left\langle r_{\perp}^{2}\right\rangle\right)$ to the rod axis at $V_{0}=70 \mathrm{kT}$. The inset shows a snapshot of the rod positions at $t=500 a_{V}^{2} / D_{\|}$. 


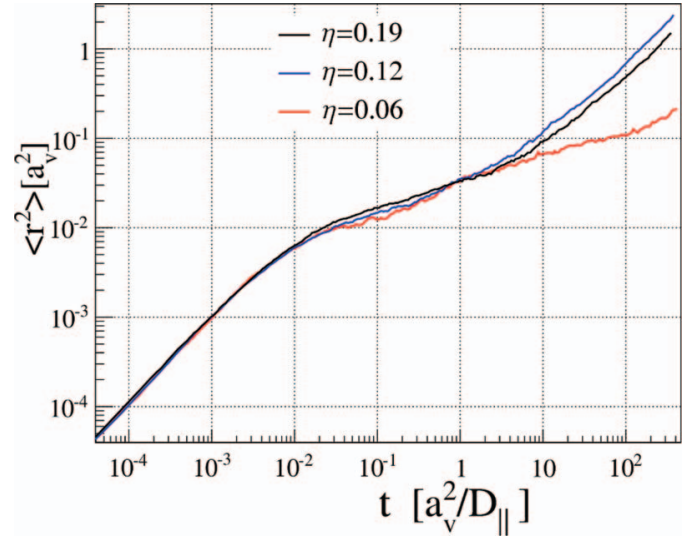

FIG. 14. Mean square displacement of a short-rod system for different area fractions at $V_{0}=70 \mathrm{kT}$.

rod axis for $V_{0}=70 k T$. Interestingly, $\left\langle\varphi^{2}\right\rangle$ and $\left\langle r_{\perp}^{2}\right\rangle$ enter the diffusive regime at the same time whereas $\left\langle r_{\|}^{2}\right\rangle$ becomes diffusive more than two decades later. The reason for this behavior is the following. Whereas one end of the rod stays trapped in its potential minimum, the other end moves/rotates into a neighboring minimum like a clock hand by thermal activation. This motion contributes to both $\left\langle\varphi^{2}\right\rangle$ and $\left\langle r_{\perp}^{2}\right\rangle$. To perform a motion parallel to the rod axis, both ends of the rod have to leave their potential minima and normal diffusion sets in later. This also explains why the total mean square displacement in (a) follows $\left\langle r_{\|}^{2}\right\rangle$.

The mobility of the short rods changes dramatically when a whole ensemble of rods is considered. In Fig. 14 we plot the mean square displacement at $V_{0}=70 \mathrm{kT}$ for different area fractions. In the dilute regime at $\eta=0.06,\left\langle r^{2}\right\rangle$ behaves similar to the single-rod system and does not reach normal diffusion within the simulation time. As soon as the rods start to cluster (region $\mathrm{C}$ in the phase diagram of Fig. 3), their mobility rises and normal diffusion is observed. The outer rods of a cluster are more weakly bound to the trap and can more easily leave their potential minima by thermal activation. Nevertheless, the diffusion coefficient is much smaller than for free diffusion by a factor of $\sim 100$. Most particles stay in their potential minima and only a few move from cluster to cluster. Further increasing the area fraction decreases the mobility again since the space between clusters becomes more and more crowded.

Compared to short rods, long rods are much more mobile when the substrate potential is switched on and the mobility is much less affected by the strength of the potential. The mean square displacement for a single long rod in Fig. 15(a) shows normal diffusion at long times even at a potential strength as high as $V_{0}=100 \mathrm{kT}$. The subdiffusive regime is always quite short. The high mobility arises from the motion of the rods along the lines of minima. They act as rails along which the rods can easily slide. To leave a rail, the rod changes its direction and occupies another rail. This picture is confirmed by Fig. 15(b). Now the accumulated mean square displacement along the rod axis, $\left\langle r_{\|}^{2}\right\rangle$, drastically exceeds $\left\langle r_{\perp}^{2}\right\rangle$ and determines translational diffusion. The ten symmetry directions for the rails are clearly visible in the snapshot of Fig. 15(b). Compared to short rods, $\left\langle\varphi^{2}\right\rangle$
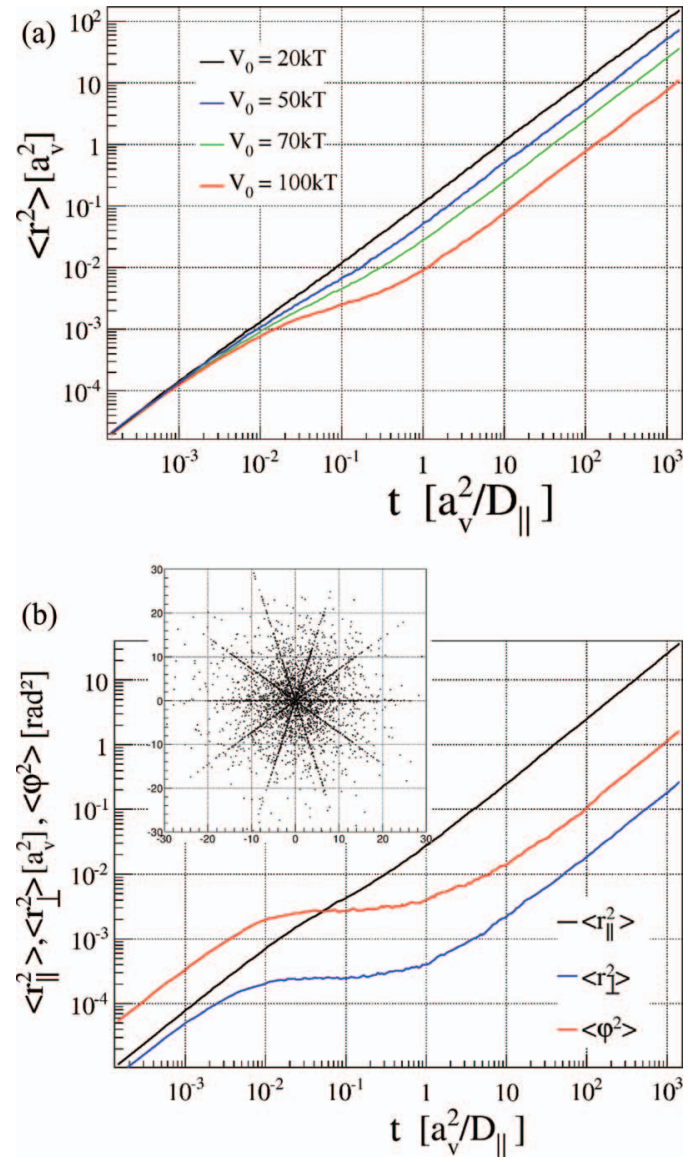

FIG. 15. (a) Mean square displacement $\left\langle r^{2}\right\rangle$ of a single long rod in units of $a_{V}^{2}$ for different potential strengths $V_{0}$. (b) Angular mean square displacement $\left\langle\varphi^{2}\right\rangle$ and accumulated mean square displacement parallel $\left(\left\langle r_{\|}^{2}\right\rangle\right)$ and perpendicular $\left(\left\langle r_{\perp}^{2}\right\rangle\right)$ to the rod axis at $V_{0}=70 \mathrm{kT}$. The inset shows a snapshot of the rod positions at $t=500 a_{V}^{2} / D_{\|}$.

is smaller by a factor of ten. In an ensemble of long rods the usual crowding occurs and in contrast to the short rods a density-induced mobility enhancement is not visible.

\section{SUMMARY AND CONCLUSIONS}

We have determined the phase behavior of hard spherocylinder systems concentrating on two rod lengths and also investigated the rod mobility.

In both systems we observe characteristic positional and directional order with decagonal symmetry that sets in when the strength of the surface potential exceeds a threshold value. Short rods connect two deep minima and orient along the symmetry directions of the substrate potential similar to short needles. ${ }^{1}$ At low area fractions this enforces directional order together with 10- and 20-fold bond-orientational order. With increasing area fraction rods form clusters. However, when the cluster size exceeds three rods, they leave the deepest minima and connect more shallow minima. This destroys directional order along the ten symmetry directions. The directional distribution function reveals a shift by $\pi / 10$ for the preferred cluster directions. For the whole range of area fractions, the pair correlation function shows pronounced positional order induced by the substrate potential. 
Long rods connect several minima and therefore slide more easily along their long axis. This results in only weak positional order and precludes any bond-orientational order for the center of mass of the rods. Pronounced directional order sets in at lower potential strength compared to the shortrod system. With increasing area fraction, long rods also cluster but due to their width they occupy separate lines of minima. Further increase of the area fraction compresses the clusters whereby the rods are pushed out of their lines of minima. Still they stay oriented along the ten symmetry directions and the directional order parameter hardly changes with increasing area fraction. When the clusters are compressed, the weak positional order vanishes completely.

The mobility of short rods decreases with increasing potential strength since they are trapped in their pair of minima. They leave this trap by rotating one end into a neighboring minimum. However, translational diffusion is determined by the hindered mobility along the rod axis. When the rods form clusters at increasing area fraction, the rod mobility increases drastically since outer rods of the clusters are more weakly bound so they leave their traps more easily. Long rods can slide along their lines of minima, therefore their mobility is much less affected by the substrate potential. Their diffusive motion is determined by the sliding while motion perpendicular to the rod axis is strongly hindered by the substrate potential.

Rods such as spherocylinders with a finite width selfassemble into novel structures under the influence of a decagonal substrate potential. They also show unexpected mobility enhancement. We hope that our theoretical investigation stimulates experimental studies using either organic molecules on surfaces of atomic quasicrystals or micrometersized rods on artificially created decagonal substrates. First theoretical results of longer hydrocarbon molecules on a quasicrystal surface have appeared recently. ${ }^{25}$ They also show the typical cluster formation as observed in our study.

\section{ACKNOWLEDGMENTS}

We thank M. Schmiedeberg and K. E. Gubbins for helpful discussions and the International Research Training Group IGRTG 1524 funded by the German Science Foundation for financial support. We also thank a referee for bringing Ref. 25 to our attention.

${ }^{1}$ P. Kählitz and H. Stark, J. Chem. Phys. 136, 174705 (2012).

${ }^{2}$ R. McGrath, J. Ledieu, E. J. Cox, and R. D. Diehl, J. Phys.: Condens. Matter 14, R119 (2002).

${ }^{3}$ V. Fournee, T. C. Cai, A. R. Ross, T. A. Lograsso, J. W. Evans, and P. A. Thiel, Phys. Rev. B 67, 033406 (2003).

${ }^{4}$ J. Ledieu, J. T. Hoeft, D. E. Reid, J. A. Smerdon, R. D. Diehl, T. A. Lograsso, A. R. Ross, and R. McGrath, Phys. Rev. Lett. 92, 135507 (2004).

${ }^{5}$ N. Ferralis, K. Pussi, E. J. Cox, M. Gierer, J. Ledieu, I. R. Fisher, C. J. Jenks, M. Lindroos, R. McGrath, and R. D. Diehl, Phys. Rev. B 69, 153404 (2004).

${ }^{6}$ M. Shimoda, T. J. Sato, A. P. Tsai, and J. Q. Guo, Phys. Rev. B 62, 11288 (2000).

${ }^{7}$ K. J. Franke, H. R. Sharma, W. Theis, P. Gille, P. Ebert, and K. H. Rieder, Phys. Rev. Lett. 89, 156104 (2002).

${ }^{8}$ R. Trasca, N. Ferralis, R. Diehl, and M. Cole, J. Phys.: Condens. Matter 16, S2911 (2004).

${ }^{9}$ S. Curtarolo, W. Setyawan, N. Ferralis, R. D. Diehl, and M. W. Cole, Phys. Rev. Lett. 95, 136104 (2005).
${ }^{10}$ W. Setyawan, N. Ferralis, R. Diehl, M. Cole, and S. Curtarolo, Phys. Rev. B 74, 125425 (2006).

${ }^{11}$ W. Setyawan, R. Diehl, N. Ferralis, M. Cole, and S. Curtarolo, J. Phys.: Condens. Matter 19, 016007 (2007).

${ }^{12}$ B. Bilki, M. Erbudak, M. Mungan, and Y. Weisskopf, Phys. Rev. B 75, 045437 (2007).

${ }^{13}$ M. Mungan, Y. Weisskopf, and M. Erbudak, Phys. Rev. B 76, 195443 (2007).

${ }^{14}$ R. D. Diehl, W. Setyawan, N. Ferralis, R. A. Trasca, M. W. Cole, and S. Curtarolo, Philos. Mag. 87, 2973 (2007).

${ }^{15}$ R. D. Diehl, N. Ferralis, K. Pussi, M. W. Cole, W. Setyawan, and S. Curtarolo, Philos. Mag. 86, 863 (2006).

${ }^{16}$ R. D. Diehl, W. Setyawan, and S. Curtarolo, J. Phys.: Condens. Matter 20, 314007 (2008)

${ }^{17}$ D. Shechtman, I. Blech, D. Gratias, and J. W. Cahn, Phys. Rev. Lett. 53, 1951 (1984).

${ }^{18}$ D. Levine and P. Steinhardt, Phys. Rev. Lett. 53, 2477 (1984).

${ }^{19}$ D. DiVincenzo and P. Steinhardt, Quasicrystals: The State of the Art, 2nd ed., Series on Directions in Condensed Matter Physics Vol. 16 (World Scientific, 1999).

${ }^{20}$ W. Steurer and S. Deloudi, Crystallography of Quasicrystals: Concepts, Methods, and Structures (Springer Verlag, 2009), Vol. 126.

${ }^{21}$ C. Janot, Quasicrystals. A Primer. (Oxford University Press, 1992).

${ }^{22}$ E. Macia, Rep. Prog. Phys. 69, 397 (2006).

${ }^{23}$ J. Y. Park, D. F. Ogletree, M. Salmeron, R. A. Ribeiro, P. C. Canfield, C. J. Jenks, and P. A. Thiel, Science 309, 1354 (2005).

${ }^{24}$ W. Setyawan, R. D. Diehl, and S. Curtarolo, Phys. Rev. Lett. 102, 055501 (2009)

${ }^{25}$ S. Curtarolo, W. Setyawan, and R. D. Diehl, Isr. J. Chem. 51, 1304 (2011).

${ }^{26}$ M. Jules, R. Johannes, H. Laurent, and B. Clemens, Nature (London) $\mathbf{4 5 4}$, 501 (2008)

${ }^{27}$ M. Schmiedeberg and H. Stark, Phys. Rev. Lett. 101, 218302 (2008).

${ }^{28}$ M. Schmiedeberg, J. Mikhael, S. Rausch, J. Roth, L. Helden, C. Bechinger, and H. Stark, Eur. Phys. J. E 32, 25 (2010).

${ }^{29}$ M. Schmiedeberg, J. Roth, and H. Stark, Phys. Rev. Lett. 97, 158304 (2006).

${ }^{30}$ J. Mikhael, M. Schmiedeberg, S. Rausch, J. Roth, H. Stark, and C. Bechinger, Proc. Natl. Acad. Sci. U.S.A. 107, 7214 (2010).

${ }^{31}$ J. Mikhael, G. Gera, T. Bohlein, and C. Bechinger, Soft Matter 7, 1352 (2011).

${ }^{32}$ C. Reichhardt and C. J. Olson Reichhardt, Phys. Rev. Lett. 106, 060603 (2011).

${ }^{33}$ M. Schmiedeberg and H. Stark, J. Phys.: Condens. Matter 24, 284101 (2012).

${ }^{34}$ M. Schmiedeberg, J. Roth, and H. Stark, Eur. Phys. J. E 24, 367 (2007).

${ }^{35}$ J. A. Kromer, M. Schmiedeberg, J. Roth, and H. Stark, Phys. Rev. Lett. 108, 218301 (2012)

${ }^{36}$ G. Oster, J. Gen. Physiol. 33, 445 (1950).

${ }^{37}$ S. Fraden, G. Maret, D. L. D. Caspar, and R. B. Meyer, Phys. Rev. Lett. 63, 2068 (1989).

${ }^{38}$ X. Wen, R. B. Meyer, and D. L. D. Caspar, Phys. Rev. Lett. 63, 2760 (1989).

${ }^{39}$ H. Graf and H. Löwen, Phys. Rev. E 59, 1932 (1999).

${ }^{40}$ Z. Zhang and J. van Duijneveldt, J. Chem. Phys. 124, 154910 (2006).

${ }^{41}$ N. Yasarawan and J. van Duijneveldt, Langmuir 24, 7184 (2008).

${ }^{42}$ P. Buining, A. Philipse, and H. Lekkerkerker, Langmuir 10, 2106 (1994).

${ }^{43}$ M. A. Bates and D. Frenkel, J. Chem. Phys. 112, 10034 (2000).

${ }^{44}$ D. Frenkel and R. Eppenga, Phys. Rev. A 31, 1776 (1985).

${ }^{45}$ J. R. Shewchuk, in Applied Computational Geometry: Towards Geometric Engineering, Lecture Notes in Computer Science Vol. 1148, edited by M. C. Lin, and D. Manocha (Springer-Verlag, Berlin, Heidelberg, 1996), pp. 203-222.

${ }^{46}$ M. Schmiedeberg, "Colloidal particles on quasicrystalline substrates," Ph.D. dissertation, TU Berlin, 2008.

${ }^{47} \mathrm{H}$. Stanley, Introduction to Phase Transitions and Critical Phenomena (Oxford University Press, 1971).

${ }^{48} \mathrm{~K}$. Binder and D. Heermann, Monte Carlo Simulation in Statistical Physics, 4th ed., Springer series in solid state sciences No. 80 (Springer, Berlin, Heidelberg, 2002).

${ }^{49}$ M. E. J. Newman and G. T. Barkema, Monte Carlo Methods in Statistical Physics (Oxford University Press, USA, 1999). 
${ }^{50}$ N. Metropolis, A. W. Rosenbluth, M. N. Rosenbluth, A. H. Teller, and E. Teller, J. Chem. Phys. 21, 1087 (1953).

${ }^{51}$ J. P. Hansen and I. McDonald, Theory of Simple Liquids (Academic, London, UK, 1990).

${ }^{52}$ K. Kikuchi, M. Yoshida, T. Maekawa, and H. Watanabe, Chem. Phys. Lett. 196, 57 (1992).
${ }^{53}$ E. Sanz and D. Marenduzzo, J. Chem. Phys. 132, 194102 (2010).

${ }^{54}$ F. Romano, C. D. Michele, D. Marenduzzo, and E. Sanz, J. Chem. Phys. 135, 124106 (2011).

${ }^{55}$ A. Patti and A. Cuetos, Phys. Rev. E 86, 011403 (2012).

${ }^{56}$ H. Löwen, Phys. Rev. E 50, 1232 (1994). 